\title{
Role of high-resolution ultrasound in the assessment of abdominal wall masses and mass-like lesions
}

\author{
Ahmed Abdelrahman Mohamed Baz ${ }^{1 *}$, Hatem Mohamed Said El-Azizi ${ }^{1}$, Mohamed Sayed Qayati Mohamed ${ }^{1,2}$ and \\ Ahmed Yehia Ibrahim Abdeldayem ${ }^{3}$
}

\begin{abstract}
Background: To evaluate the diagnostic accuracy of high-resolution ultrasound in the assessment of abdominal wall masses and mass-like lesions and to provide an overview of the sonographic appearances of different abdominal wall pathologies, 54 patients were included in a prospective study. The patients' age was ranging from 1 to 75 years. Twenty-eight were females (51.9\%) and 26 were males (48.1\%); all were evaluated by a high-resolution ultrasound examination, and the results were correlated to the patients' operative findings and histopathological results as well as the pelviabdominal $C T$ findings.

Results: In comparison to the operative, histopathological, and CT findings, the high-resolution ultrasound had an overall 100\% accuracy for abdominal wall lesions, and for hernia cases, it had 100\% sensitivity, 100\% specificity, 100\% accuracy, 100\% positive predictive value, and 100\% negative predictive value.

Conclusion: The current study had encouraging results regarding the role of high-resolution ultrasound in the assessment of abdominal wall masses and mass-like lesions; nevertheless, it is considered as an effective and simple diagnostic tool that may limit the patients' exposure to invasive biopsies and to the hazardous exposure to ionizing radiation and contrast media administration like that in $\subset$ examination.
\end{abstract}

\section{Background}

The abdominal wall is an anatomical term used to describe the layers that cover the abdominal cavity from its anterior, posterior, and lateral aspects. It consists mainly of skin, subcutaneous fat, fascia, and a muscle layer as well as the parietal peritoneum. As the anterior and the lateral walls have no definite boundary, thus, both could be described collectively as the anterolateral wall. $[1,2]$.

The anterolateral abdominal wall encompasses two groups of paired muscles, those are the vertical recti abdominus muscles as a medical group and the flat (sheet) like muscles of the flanks including from exterior to interior, the external oblique, the internal oblique, and the transversus abdominus muscles (Fig. 1) $[1,2]$.

\footnotetext{
* Correspondence: ahmedbaz2012@yahoo.com

${ }^{1}$ Radiology Department, Faculty of Medicine, Cairo University, Giza, Egypt Full list of author information is available at the end of the article
}

The recti abdominus muscles are paired muscles that are separated in the midline by the linea alba and are completely enclosed by the rectus sheath except for the posterior surface of the lower quarter. The three flat muscles are arranged in three overlying layers in which the external oblique muscle is the most superficial one and the transversus abdominus is the deepest one; the three muscles had three separate but layered aponeuroses that have insertions in the costal margin and in the linea alba. The lower margin of the external oblique muscle aponeurosis forms the inguinal ligament that contributes to the inguinal canal formation $[1,2]$.

The inguinal canal is a slit-like space that starts at the deep inguinal ring located just lateral to the inferior epigastric artery and ends in the superficial inguinal ring that is located superior to the pubic tubercle and contains the spermatic cord in males and the round ligament in females (Fig. 2) [1, 2]. 
The posterior abdominal wall is formed by the spine medially and by the psoas muscle that arises from the spine and joins the iliacus muscle to form the iliopsoas tendon to be inserted at the lesser trochanter of the femur and the quadratus lumborum muscle that extends from the last rib to the iliac crest (Fig. 3) [3].

High-resolution (high-frequency) ultrasound that may be complemented by a low-frequency (deep) probe for deeper structures and thick abdominal walls had been widely used as an accepted diagnostic tool for evaluation of the abdominal wall pathologies including hernias, masses, and mass-like lesions [4].

Being available, cheap, and lacking the exposure to ionizing radiation, the ultrasound had gained a wide range of usage; moreover, it permits the assessment of the patient while performing dynamic maneuvers like Valsalva and examination in the upright position. The extended field of view (panoramic view) had also added value for better delineation of the anatomical relationship of the lesions. Color and power Doppler images are useful in assessing the lesion vascularity and the relationship of the lesion to the nearby important vascular structures [4].

On ultrasound examination, the subcutaneous fat appears hypoechoic next to the echogenic skin layer while the muscle layer appears as pinnate-like or branching echogenic septae in between the hypoechoic muscle fibers. The parietal peritoneum appears as a continuous echogenic line next to the hypoechoic thin transversalis fascia [5].

The abdominal wall lumps have a wide range of differential diagnosis by the clinical examination; unfortunately, the physical findings often lack the specificity and a confirmatory study may be warranted especially if surgical intervention is planned.

A spectrum of the abdominal wall lesions had been tabulated in Table 1 with a brief description of the ultrasound features of each lesion in order to simplify and to narrow the range of differential diagnosis for the examiners.

\section{Aim of work}

The aim of this work was to evaluate the usefulness of performing high-resolution ultrasound in the assessment of abdominal wall masses and mass-like lesions and to provide an overview of the sonographic appearances of different abdominal wall pathologies.

\section{Methods}

Subjects

Fifty-four patients were included in the present study; all were recruited from the General Surgery outpatient clinic in our institute, during the period from August 2018 to February 2019. The patient's age was ranging from 1 to 75 years (mean age $=40.76$ years). Twenty-eight were females and 26 were males.

Inclusion criteria. All patients presenting with an abdominal wall swellings were included in the current study.

Exclusion criteria. The patients who had not the willingness to participate in the study were excluded.

The protocol was reviewed and approved by the local ethics committee.

\section{Methods}

Our patients were subjected to:

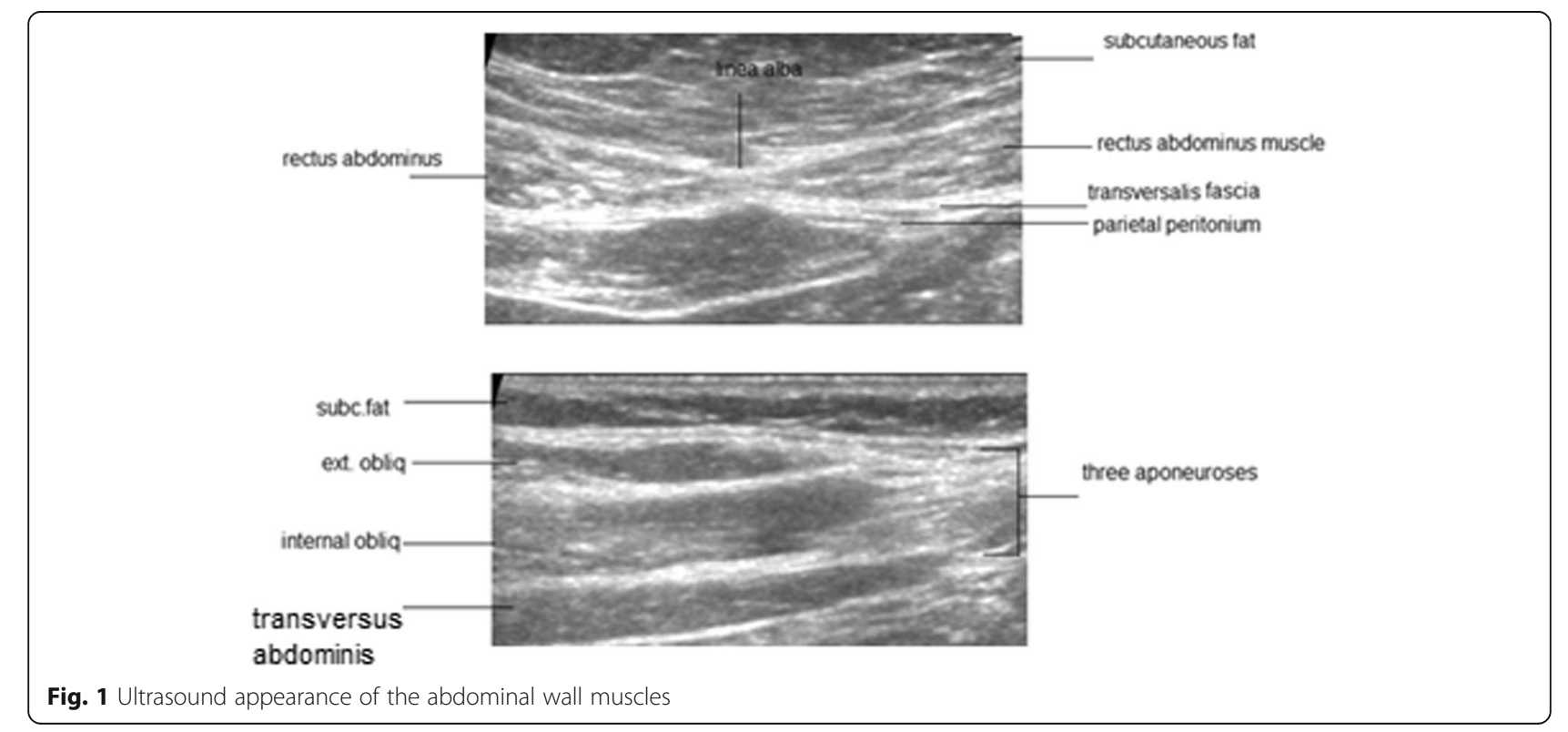




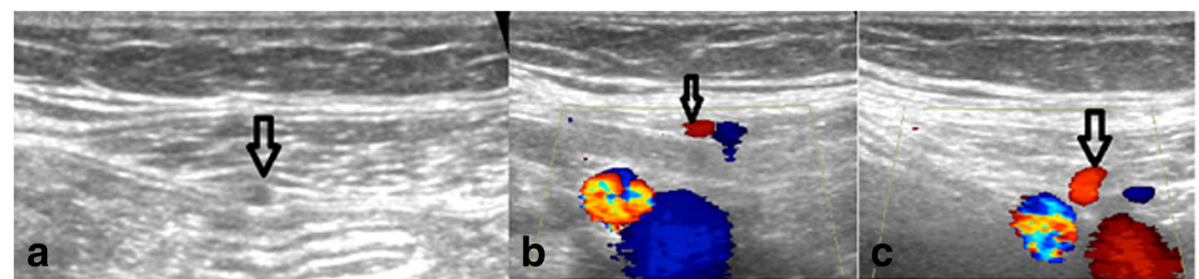

Fig. 2 The ultrasound (a) and color Doppler images (b, c) identifying the inferior epigastric artery (black open arrows)

1. Detailed history taking in which every patient had to answer the following questions:

a. When did the swelling start to appear?

b. Was there any history of other associated abdominal complaints?

c. Was there any history of previous abdominal surgeries?

d. Whether there were any planned abdominal surgeries?

\section{Examination by high-resolution ultrasound}

All patients had done focused abdominal wall examination by high-resolution ultrasound.

Focused abdominal wall ultrasound was done using Toshiba Aplio 500, General Electric Logic P7, General Electric Logic S8, and General Electric Voluson E6; all examinations were done by a linear probe of $(7.5-12 \mathrm{MHz}$ frequency) and sometimes by a curvilinear probe of (3.5$5 \mathrm{MHz}$ ) for thick abdominal walls and for examination of deeper structures beyond the depth of the linear probe.

The examination was done by a radiology resident under the supervision of an expert radiologist who had 12 years of experience in different ultrasound examinations and supervised all the examination steps for all cases in the study.

The examination steps were explained in details for the patients, and the patients were trained enough for the dynamic maneuver performance.
A manual reduction of a hernia was done by asking the patient himself to reduce it if he could do this, but if he could not, the examiner asked the patient to lay down, then the hernia could be reduced spontaneously or by simple manipulations. Most of the examined hernias were totally or partially reducible; irreducibility is a sign of complication, either strangulation or incarceration, and this was only seen in one patient in our study.

Extended or panoramic views were occasionally taken to demonstrate the relationship between the lesion and the nearby structures in the abdominal wall. There were no needs for any abdominal preparation. Any abdominal wounds were cleaned and, if possible, covered by a thin, sterile, plastic adhesive membrane, or, more simply, the probe was covered by a sterile glove. The patients lied supine while their lower extremities were extended.

The gel (matching medium) was applied to the ultrasound probe and preferably to be warmed. A targeted ultrasound examination was done on the site of the patient complaint. A multiplanar scanning was done. Color or power Doppler imaging was used to assess the vascularity of the mass or mass-like lesions and in order to identify the relationship of the lesion to the regional important vessels.

For hernias, the hernia defect widths were measured and their contents were assessed. In inguinal hernia cases, we tried to first identify the spermatic cord at the level of the external ring then the cord was followed laterally until we reached a level where it sinks into the peritoneal cavity through the deep inguinal ring; the latter could be identified by its

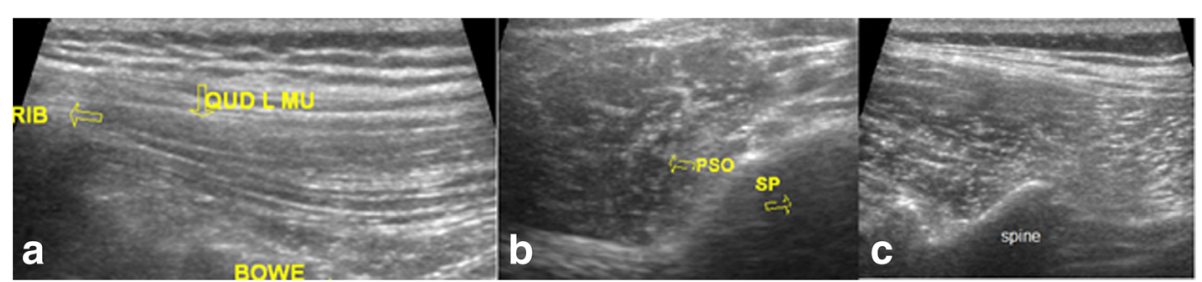

Fig. 3 The ultrasound images (a) for quadratus lumborum muscle and for the psoas muscle as it arises from the spine in a transverse section (b) and in a longitudinal section in $\mathrm{c}$ 
Table 1 A spectrum of the abdominal wall lesions with the demonstration of their ultrasound features $[4-6,9,11]$

\begin{tabular}{|c|c|}
\hline Lesion & Ultrasound features \\
\hline $\begin{array}{l}\text { 1. Hernia } \\
\text { a. Inguinal }\end{array}$ & $\begin{array}{l}\text { An abdominal viscus, commonly the bowel loops or omentum protrudes through a defect; the location of the defect in } \\
\text { relation to the inferior epigastric artery determines the type of hernia; thus, a defect located medial to the artery makes the } \\
\text { diagnosis of a direct hernia and vice versa. }\end{array}$ \\
\hline b. Ventral & $\begin{array}{l}\text { Including the supra-umbilical, umbilical, and infra-umbilical hernias where a defect is present and the omental fat or the } \\
\text { bowel protrude through it. }\end{array}$ \\
\hline c. Incisional & Where a defect is present at the site of previous surgical scarring. \\
\hline d. Recurrent & Where a hernia reoccurs at the same site of a previous hernia repair. \\
\hline 2. Lipoma & $\begin{array}{l}\text { Rather circumscribed hypo or hyperechoic soft tissue swelling with different shapes but commonly ovoid or spindle shape } \\
\text { with interlacing echogenic fibrous bands giving a feathery appearance. }\end{array}$ \\
\hline 3. Endometrioma & $\begin{array}{l}\text { Often following abdominal surgery (e.g., Cesarean section) and usually present as a palpable lump that is associated with } \\
\text { menstrual cycles and sonographically has different shapes varying from simple cystic, complex cystic, and even solid mass- } \\
\text { like lesions. }\end{array}$ \\
\hline 4. Hematoma & $\begin{array}{l}\text { The patient has a history of anticoagulation or vigorous exercise that provokes the vascular rupture of either the superior or } \\
\text { inferior epigastric artery; on ultrasound, a recent hematoma has a rather inhomogeneous echogenic pattern with possible } \\
\text { layering fluid or fluid-fluid levels, but older hematomas tend to be absorbed or to liquefy giving a cystic appearance. }\end{array}$ \\
\hline 5. Seroma & $\begin{array}{l}\text { Is a postoperative sequel and appears as a cyst-like lesion with acoustic enhancement but no solid components could be } \\
\text { found inside. }\end{array}$ \\
\hline 6. Abscess & $\begin{array}{l}\text { Is a complex fluid collection with debris and low to medium level internal echoes; it may be related to a surgical wound } \\
\text { where it is called a suture abscess and the echogenic sutures could be identified in many cases; however, it may be difficult } \\
\text { to differentiate the abscess from hematoma and seroma based on the ultrasound picture, but irregular walls, marginal } \\
\text { vascularity, and the surrounding inflammatory changes may help to differentiate, but sometimes, the only feasible way of } \\
\text { differentiation is to do an aspiration. }\end{array}$ \\
\hline 7. Metastasis & $\begin{array}{l}\text { It may occur by a lymphatic spread or blood-borne tumor dissemination. Melanoma is common but other tumors like } \\
\text { bronchogenic carcinoma or lymphoma may be also considered; on ultrasound, it appears as an ill-defined hypoechoic mass } \\
\text { with increased internal vascularity. }\end{array}$ \\
\hline $\begin{array}{l}\text { 8. Neurogenic } \\
\text { tumors }\end{array}$ & $\begin{array}{l}\text { Either schwannoma or neurofibroma, by ultrasound, both appears as well-circumscribed fusiform or ovoid swellings with } \\
\text { tapering ends to the parent nerve. }\end{array}$ \\
\hline $\begin{array}{l}\text { 9. Vascular } \\
\text { malformations }\end{array}$ & $\begin{array}{l}\text { It could be either a low flow or a high flow lesions where multiple intercommunicating cystic spaces are found and may } \\
\text { show by color Doppler either a pure venous flow (low flow type) or evidence of arteriovenous shunting (high flow); } \\
\text { sometimes, calcific phlebolith may be seen. }\end{array}$ \\
\hline 10. Desmoid tumor & $\begin{array}{l}\text { Is a myofibroblastic neoplasm that is considered as deep fibromatosis and often follows abdominal surgery or abdominal } \\
\text { wall scarring. Gardner's syndrome and estrogen therapy may be considered as risk factors; it appears as a poorly } \\
\text { circumscribed hypoechoic soft tissue lesion that infiltrates the abdominal wall muscles and may present internal vascularity, } \\
\text { and characteristically, the lesion does not cross the midline. }\end{array}$ \\
\hline
\end{tabular}

location just lateral to the inferior epigastric artery. If no hernia had been identified, then the straining maneuvers like Valsalva or performing the examination while the patient in the standing position was done alternatively. If a hernia was present, then the relationship of the hernia sac defect to the inferior epigastric vessels was established to determine whether it was an indirect or a direct type. A similar technique was used in the female patients by identifying the round ligament in the suprapubic region close to the labia majora and following it laterally in a similar fashion. Color and power Doppler imaging were helpful in both males and females in order to identify the spermatic cord and the round ligament where they were more readily depicted by their accompanying vessels in addition to the assessment of the vascularity of their contents.

For umbilical hernias, the same straining maneuvers that were used for the detection of the inguinal hernias were also used in addition to raising the patients' head while they were laying down and had also helped in divarication of the recti abdominus muscles and hence for the appreciation of the presence of a hernia sac.

For any mass lesion, the size, site, and the echogenicity pattern were assessed in addition to the internal vascularity of the lesion as well as the relationship to any important structure in multiple orthogonal scanning planes. Similar steps were followed for the assessment of any cystic lesion in addition to manual compression by the probe to identify the lesion compressibility and if there was any deep connection to the abdominal cavity.

\section{Statistical methods and data analysis}

Statistical analysis was performed using Statistical Package for the Social Sciences (SPSS version 23) software. The results were expressed as mean, frequencies, or 
Table 2 The clinical diagnosis of the abdominal wall masses and the mass-like lesions with the demonstration of the number (no.) and the percent (\%) of the affected patients

\begin{tabular}{lll}
\hline Clinical diagnosis & Number of patients (no.) & Valid percent (\%) \\
\hline Hernia & 32 & 59.2 \\
Non-specified lump & 12 & 22.2 \\
Abscess & 4 & 7.4 \\
Lipoma & 2 & 3.7 \\
Neurofibromatosis & 1 & 1.9 \\
Postoperative seroma & 1 & 1.9 \\
Hematoma & 1 & 1.9 \\
Hemangioma & 1 & 1.9 \\
\hline
\end{tabular}

relative frequencies. The data were collected, analyzed, and tabulated. The sensitivity, specificity, positive predictive value, negative predictive value, and the diagnostic value of the high-resolution ultrasound in the diagnosis of abdominal wall masses and mass-like lesions were calculated using the standard definitions.

\section{Results}

Fifty-four patients were enrolled in the present study. The patients' age was ranging from 1 to 75 years. Twenty-eight were females (51.9\%) and 26 were males (48.1\%).

All patients presented with abdominal wall swelling with different clinical diagnoses. Thirty-two patients were clinically diagnosed as hernias (59.2\%), of which 14 were groin (inguinal) hernias, nine were paraumbilical, six were umbilical, and three were incisional hernias. Twelve patients were diagnosed clinically as a non-specified lump (22.2\%).Two patients presented with a clinical diagnosis of lipoma (3.7\%). One patient was presenting with a clinically suspected neurofibroma (1.9\%). Five patients presented with a postoperative anterior abdominal wall fluctuant swelling of which four (7.4\%) had been suspected clinically to have an abscess, and one patient (1.9\%) was suspected to have a recurrent hernia versus a postoperative collection. One patient (1.9\%) was on oral anticoagulants and had been suspected clinically to have a rectus sheath hematoma (1.9\%). One patient (1.9\%) presented with a clinical diagnosis of hemangioma (Table 2).

Fourteen patients (11 females and three males) had a history of previous abdominal operations (25.92\%), of which seven patients had done Cesarean sections, two patients had a hernia repair, two patients had done intestinal resection and anastomosis, one patient had an appendectomy, one patient had a cholecystectomy, and one patient had done surgical evacuation of a post-traumatic rectus muscle hematoma.

On examination of the abdominal wall lesions by a high resolution ultrasound, the following was depicted: 31 patients had a hernia (57.4\%) (Fig. 4), 13 patients of them had an inguinal hernia, nine patients had a paraumbilical (epigastric or hypogastric) hernia, six patients had an umbilical hernia, two patients had an incisional hernia, and one patient had a Spigelian hernia. Six patients had abscesses (11.1\%) (Fig. 5), two of them had suture abscesses, one patient had foreign body granuloma with abscess formation and one patient had a cold abscess, and the other two abscesses showed no suture material inside them (nonsuture abscess). Four patients had endometriomas (7.4\%) (Fig. 6), four patients had a desmoid tumor (7.4\%) (Fig. 7), and two patients had a lipoma (3.7\%), one at the anterior abdominal wall and one at the posterior abdominal wall. Two patients had a hematoma (3.7\%) (Fig. 8), of which one was in the inguinal region. Five patients had different diagnoses including neurofibromatosis (1.9\%), postoperative seroma (1.9\%) (Fig. 5), venous vascular malformation (1.9\%), abdominal wall metastasis (1.9\%) (Fig. 9), and hydrocele of the canal of Nück (1.9\%) (Table 3).

Three correlative studies had been done for comparison to the high-resolution ultrasound findings including the following: 50 patients (27 females and 23 males) had been operated upon for their abdominal

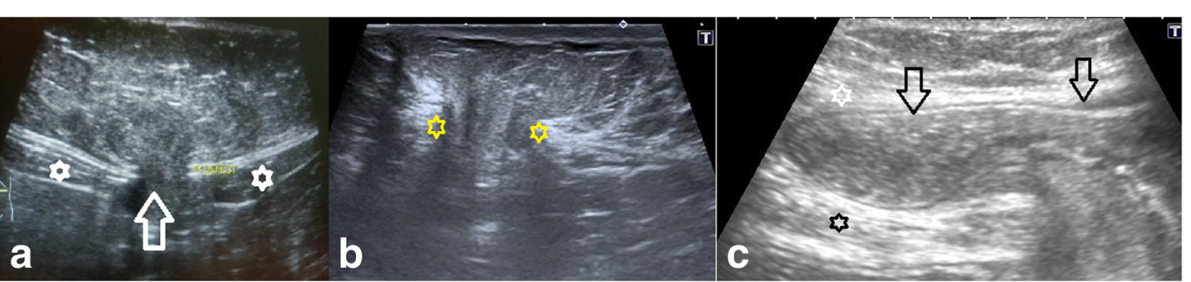

Fig. 4 a-c Grayscale ultrasound images for three patients presenting with an anterior abdominal wall swellings. a An image for a case of fatty hernia of the linea alba showing a midline defect in the linea alba (white open arrow) between the two recti abdominus muscles (white asterisks) with the abdominal fat being seen protruding through the defect in a mushroom-like configuration. b An incisional hernia with a defect (between the yellow asterisks) showing protrusion of the intra-abdominal fat through it. c An image for a case of Spigelian hernia with a bowel loop (open black arrows) being seen protruding through an abdominal wall defect to be insinuated between the transversus abdominus (black asterisk) and the internal oblique (white asterisk) muscles. In the three cases, findings were operatively confirmed 


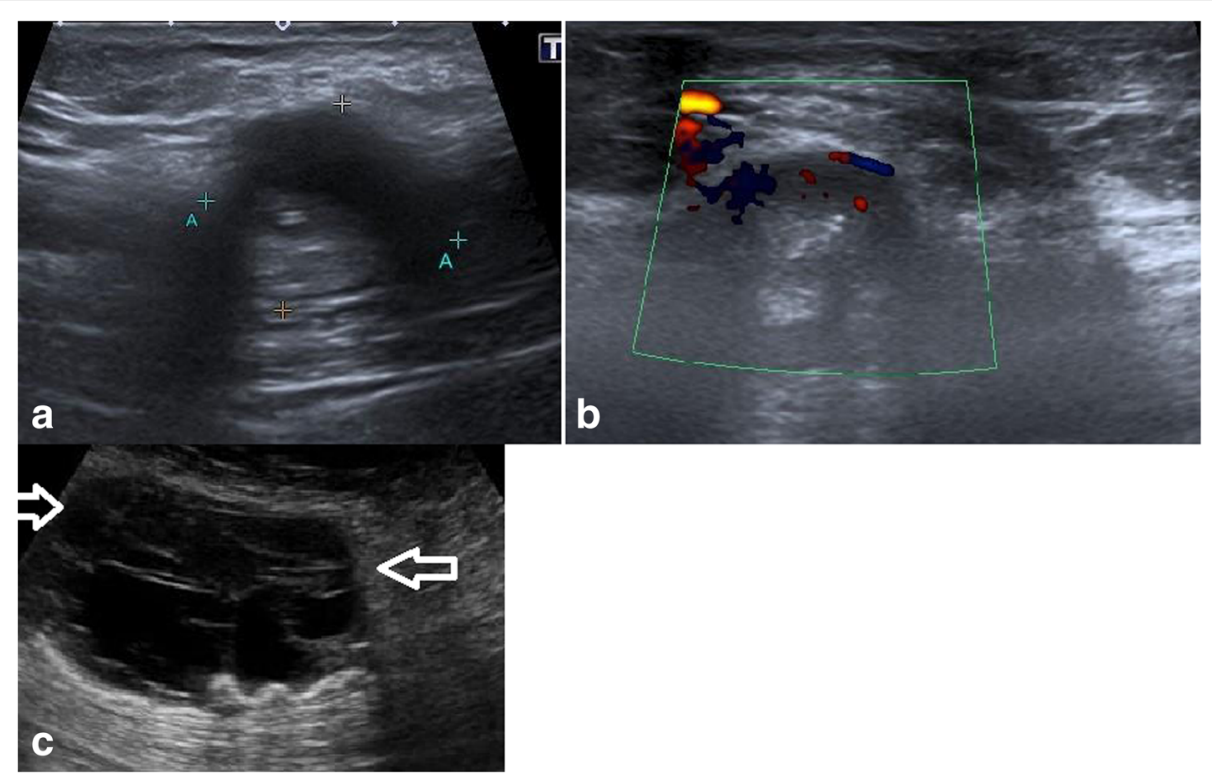

Fig. 5 a-c Ultrasound images for two different patients presenting with an anterior abdominal wall postoperative fluctuant swellings. a, b Ultrasound and color Doppler images for a patient with a right iliac fossa swelling showing hypoechoic subcutaneous collection with double echogenic suture fragments inside (curved white arrow in a) and peripheral vascularity (marginal vascularity) is seen in b; this was operatively confirmed as a suture abscess. c An ultrasound image for another patient showing a large septated supraumbilical abdominal wall subcutaneous collection (between the white open arrows) that was present after a mesh placement for an epigastric hernia repair; this was operatively proven as a postoperative seroma, and a hernia recurrence was excluded

wall lesions (92.6\%) (32 cases with hernia, 6 with abscesses, 4with desmoid, 4 with endometriomas, 2 with lipoma, 1 with hydrocele of canal of Nück, and 1with a hematoma), 13 patients (nine females and four males) (24.1\%) had done biopsies for their evaluation, and eight patients (five females and three males) had done pelviabdominal CT scans (14.8\%) for further assessment of their abdominal wall lesions.

Histopathology correlation was done for all excised masses and for the trucut biopsies cases (13 cases) including 4 cases with desmoids tumor, 4 cases with endometriosis, two cases with lipoma, one case with

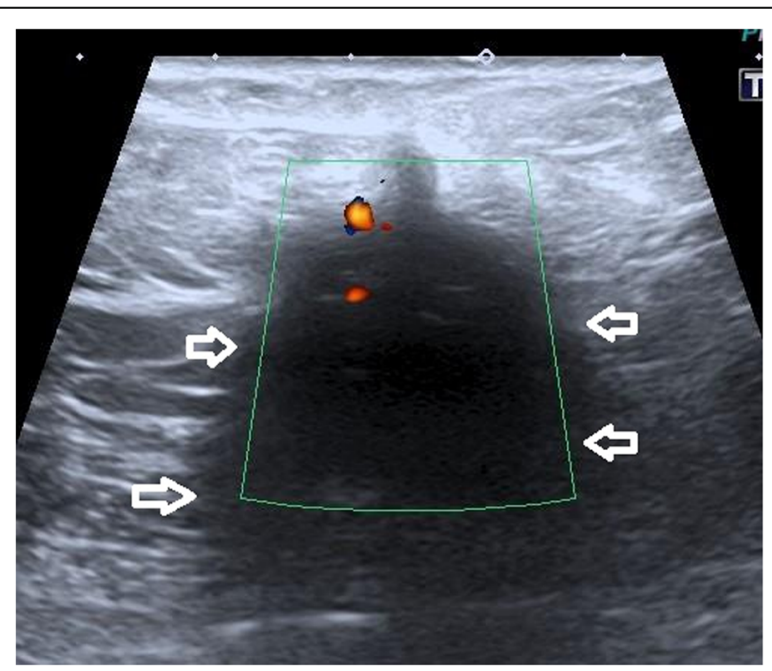

Fig. 6 A color Doppler image showing a hypoechoic left rectus abdominus muscle lesion (between the open white arrows) with peripheral vascularity for a 32-year-old female presenting left para-umbilical swelling that was histopathologically confirmed as an abdominal wall muscle endometrioma 

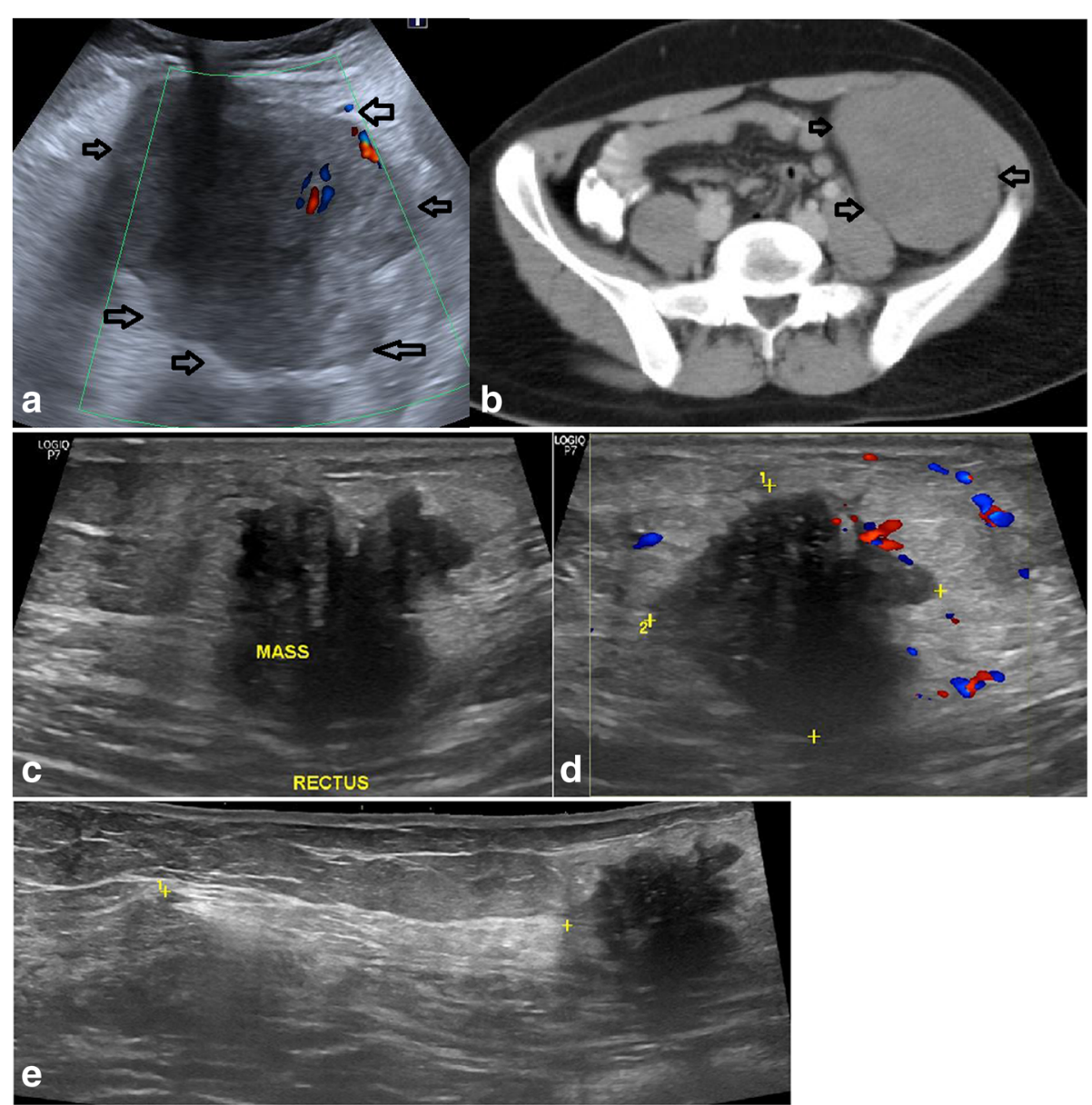

Fig. 7 a A color Doppler image showing a hypoechoic mass lesion infiltrating the abdominal wall muscles (between the open black arrows). b An axial post-contrast CT image of the lower abdomen for the same patient showing an isodense lesion (between the black arrows) infiltrating the left anterior abdominal wall muscles; this was histopathologically proven to be a desmoid tumor. c-e Ultrasound images for another patient with a left rectus abdominus muscle desmoids tumor: $\mathrm{c}$ an ultrasound image showing a lobulated hypoechoic lesion infiltrating the left rectus abdominus muscle, $\mathrm{d}$ a color Doppler image showing peripheral and peri lesion vascularity, e a panoramic ultrasound view demonstrating the hypoechoic infiltrating lesion infiltrating the lower-left rectus abdominus muscle (between the calibers)

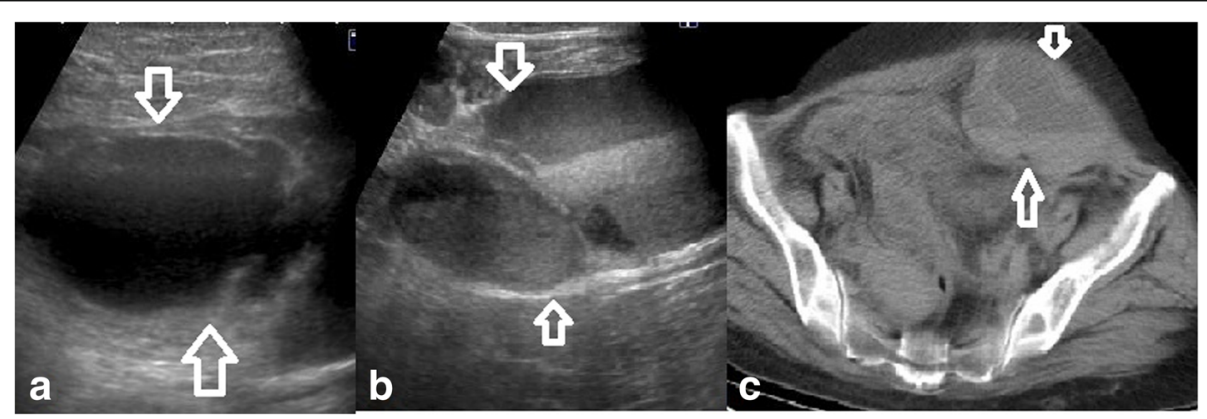

Fig. 8 a-c Ultrasound and CT images for a case of rectus sheath hematoma. a An ultrasound image showing anechoic (cyst-like) lesion implicating the left rectus abdominus muscle (between the white arrows). b An ultrasound image for the same patient at a lower level showing internal septations with layering low-level internal echoes, and possible fluid levels were seen likely due to cellular sedimentation (between the white arrows). c An axial CT image confirming the findings that were present in a and b 


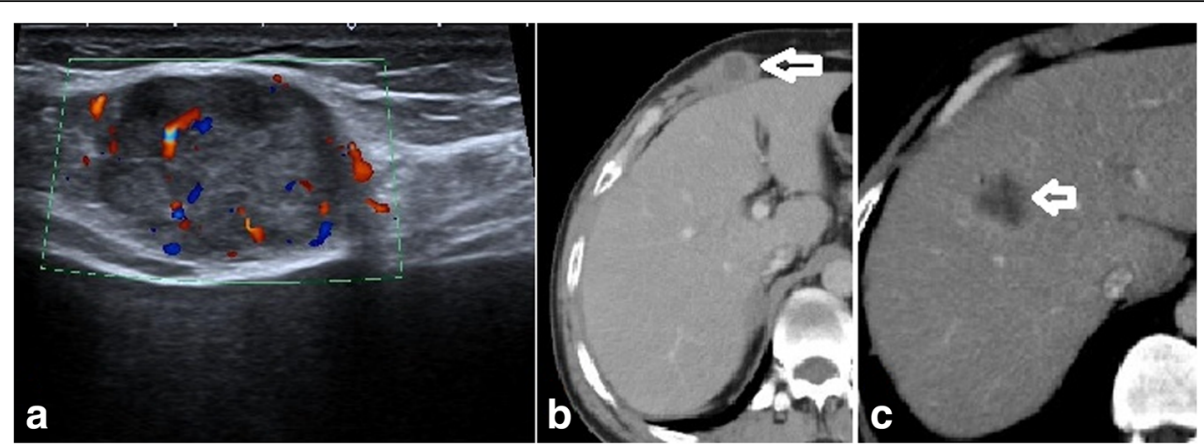

Fig. 9 a A color Doppler image showing a hypoechoic mass lesion infiltrating the abdominal wall muscles and showing internal vascularity. b An axial post-contrast CT image of the upper abdomen for the same patient showing a hypodense lesion (open white arrow) infiltrating the right rectus abdominus muscle. c An axial post-contrast CT image of the upper abdomen for the same patient showing a hypodense right hepatic lobe heterogeneously enhancing focal lesion that was incidentally found (open white arrow); this was histopathologically proven as a right rectus muscle metastatic nodule for a primary bronchogenic carcinoma

neurofibroma, and one case with abdominal wall metastasis; a case with cold abscess was drained with cytological analysis

CT was done for 7 cases ( two cases with desmoids tumor, one case with metastatic deposits, one case with venous malformation, one case with an abscess, one case with Spigelian hernia, and one case with a hematoma; the latter was treated conservatively and resolved spontaneously with no intervention).

One case was diagnosed as hemangioma and, actually, the patient had another hemangioma in his face (diagnosed by MRI), and both were treated by injection sclerotherapy.

In comparison to the operative findings, histopathological results, and the pelviabdominal CT findings, the accuracy of the high-resolution ultrasound in the diagnosis of all abdominal wall lesions was $100 \%$. For the hernia cases, the ultrasound had 100\% sensitivity, $100 \%$ specificity, $100 \%$ accuracy, $100 \%$ positive predictive value, and $100 \%$ negative predictive value (Table 4).

\section{Discussion}

The abdominal wall lesions had been identified as a very common entity that could be seen in the clinical and radiological examinations. Nevertheless, the clinical findings in the abdominal wall lesions often had a low specificity and in many situations, and the clinically suspected intra-abdominal masses might be surprisingly arising from the abdominal wall [5].

The imaging workup of the abdominal wall lesions could be performed by different modalities; however, the ultrasound had predominated in this regard, being widely available, noninvasive, and of low cost; moreover, it provides a high spatial resolution of the abdominal wall layers. The real-time examination obtained through the ultrasound gives the opportunity for assessing the patient while performing the straining maneuvers as well as in the standing position if there was an indication for this $[4,6,7]$.

The current study included 54 patients, 28 were females $(51.9 \%)$ and 26 were males (48.1\%); thus, we

Table 3 The results by the high-resolution ultrasound examination with the demonstration of the number (no.) and the percent (\%) of the affected patients

\begin{tabular}{lll}
\hline High-resolution ultrasound diagnosis & Number of patients (no.) & Valid percent (\%) \\
\hline Hernia & 31 & 57.4 \\
Desmoid & 4 & 7.4 \\
Endometriosis & 4 & 7.4 \\
Abscess & 6 & 11.1 \\
Lipoma & 2 & 3.7 \\
Neurofibromatosis & 1 & 1.9 \\
Postoperative seroma & 1 & 1.9 \\
Hematoma & 2 & 3.7 \\
Right inguinal hydrocele of the canal of Nück & 1 & 1.9 \\
Right rectus abdominus muscle metastasis & 1 & 1.9 \\
Venous malformation & 1 & 1.9 \\
\hline
\end{tabular}


Table 4 The accuracy measures for the high-resolution ultrasound diagnosis

\begin{tabular}{lll}
\hline Accuracy measure & Value (\%) & 95\% confidence interval (Cl) \\
\hline Sensitivity & 100.00 & 88.78 to $100.00 \%$ \\
Specificity & 100.00 & 85.18 to $100.00 \%$ \\
Positive predictive value & 100.00 & \\
Negative predictive value & 100.00 & \\
Overall accuracy & 100.00 & 93.40 to $100.00 \%$ \\
\hline
\end{tabular}

had the same gender distribution as Jayaram et al., Babu et al., and Devareddy et al., who also had studied abdominal wall lesions by high-resolution ultrasound examination [7-9].

Babu et al. conducted a research in 2018 to evaluate the role of high-resolution ultrasound in the evaluation of the anterior abdominal wall lesions. Their study included 50 patients (66.7\% females and $33.3 \%$ males) [8].

Another study was done by Jayaram et al. to evaluate the role of ultrasound scanning in the diagnosis of equivocal ventral hernias in comparison to the operative findings, and their study was larger with a total of 348 patients $(56.9 \%$ females and $43.1 \%$ males) included [7].

We agreed with Babu et al. in their explanation for the female gender predominance as the Caesarean section and the abdominal hysterectomies are considered as the most common surgical procedures that are performed worldwide [8].

In our study, a hernia was the most frequently encountered abdominal wall lesion (57.4\%). These results were consistent with Babu et al., who had the same results (58\% of their patients) [8].

Devareddy et al. had also reported that a hernia was the most frequently encountered abdominal wall lesion (70\%), and this was also concordant with our results [9].

In our study, the age of hernia patients was in the range of 20-50 years, and this went with the studies done by Babu et al. and Devareddy et al.; however, one of our cases showed a younger age (1-year-old female), and it was clinically diagnosed as a congenital inguinal hernia, but the ultrasound examination revealed a hydrocele of canal of Nück.

Regarding the type of a hernia, the current study showed that the predominant type of hernia was the ventral hernia $(48.3 \%)$ followed by the inguinal hernia $(38.7 \%)$, then the incisional hernia (9.6\%), and Spigelian hernia (3.2\%); this was discordant with the results reported by Babu et al. who had documented that the incisional hernia was the frequent one $(44 \%)$ followed by the ventral type (14\%), and this could be explained by the small sample size in both studies (our study was conducted on 54 patients while theirs was on 50 patients); thus, a small sample size may explain this difference, and a larger sample size may be required for a reproducible and more representative data [8].

Discordance with Devareddy et al.'s study had been noted, who had reported that the incisional hernia (56\%) was the most common type followed by the ventral hernia (14\%) [9]; this difference may be explained by a relatively low percentage of postoperative patients (25.9\%) (who had a previous history of old operations as a predisposing factor for incisional hernia) among the cases of our study compared to theirs, where they were concerned with the abdominal wall lesions in the postoperative patients.

There is an agreement with the study done by ElSayed et al., who conducted their study on 50 patients and found that a ventral hernia was the most common abdominal wall lesion; they had emphasized on the important role of the ultrasound in the hernia evaluation, especially if there were any suspected complication, and highlighted its role as a confirmatory diagnostic tool when the clinical and other imaging studies were inconclusive [10].

In the present study, we had only one case of complicated hernia that was a strangulated one, and this was confirmed intra-operatively; we can explain this as these complications are surgical emergencies and usually the patients seek the medical advice at the ER and immediate exploration and reduction are to be done urgently, but our patients were mostly referred from the outpatient clinics and rarely from the ER if ever.

Usually, the clinical diagnosis of a hernia is correct and this was seen in our study where 32 cases were diagnosed as a hernia by the clinical examination, but the ultrasound examination revealed only 31 cases, and this discrepancy was in only one case; it was diagnosed clinically as a postoperative recurrent hernia, but the ultrasound examination revealed a postoperative seroma that was surgically evacuated, and this could enhance the points of strength of the role of ultrasound examination (Fig. 5).

Regarding the accuracy measures for the high-resolution ultrasound validity in the diagnosis of the abdominal wall and groin hernias compared to the operative, histopathology, and CT findings, the current study had a sensitivity of $100 \%$, specificity $100 \%$, a positive predictive value of $100 \%$, and a negative predictive value of $100 \%$ with a diagnostic accuracy of $100 \%$.

There is a slight difference with Jayaram et al. who had reported sensitivity of $91.8 \%$, specificity of $80 \%$, and positive predictive value of $97.8 \%$ [7] and with Devareddy et al. who had reported a sensitivity of $100 \%$, specificity of $75 \%$, a positive predictive value of $97.4 \%$, a negative predictive value of $100 \%$, and a 
diagnostic accuracy of 97.6\% [9]; however, Babu et al. had also reported a diagnostic accuracy of $97.6 \%$ [8].

This difference could be attributed to the limited number of patients in our study that had included 54 patients and the different correlative modalities where we used the operative, histopathological, and the CT findings as gold standards while their studies had used only the operative and the biopsy findings.

However, there was an agreement by these different studies [7-10] that the high-resolution ultrasound had encouraging results regarding its role in the assessment of abdominal wall masses and mass-like lesions with an accuracy of over $97 \%$.

We had some limitation in the current study: the first one was the limited number of patients since we conducted our study on 54 patients only, and the second one was the relatively low percentage of postoperative patients $(25.9 \%)$ who are at risk for incisional hernia. So, further research with a large number of patients and including a higher proportion of postoperative patients is considered.

\section{Conclusion}

This study had encouraging results regarding the role of high-resolution ultrasound in the assessment of abdominal wall masses and mass-like lesions; nevertheless, it is considered as an effective and simple diagnostic tool that may limit the patients' exposure to invasive biopsies and to the hazardous exposure to ionizing radiation and contrast media administration like that in the CT examination.

\section{Abbreviations}

CT: Computed tomography; ER: Emergency room; HRUS: High-resolution ultrasound; MRI: Magnetic resonance imaging

\section{Acknowledgements}

Not applicable, as all authors are co-authors for this research.

\section{Authors' contributions}

$A A M B$ is the corresponding author who had contributed to the direct supervision of the ultrasound examinations and the interpretation of the corresponding correlative image studies done in the research work. HMSE-A had introduced the idea of the current study and helped in the image selection and in the editing of the manuscript and reference collection. MSQM had done the ultrasound examination, data collection, and follow-up of the operative data and the histopathological data as well. He also shared in the editing and in the revision of the manuscript. AYIA contributed to the selection of patients with the inclusion criteria, operative intervention, and the clinical assessment of the patients. All authors read and approved the final manuscript.

\section{Funding}

All authors had no fund for this research and had no competing interests.

\section{Availability of data and materials}

All data are available on a software system owned by each of the authors, and the corresponding author has the authority to respond if there is any query.

\section{Competing interest}

The authors declare that they have no competing interests.
Ethics approval and consent to participate

The protocol was reviewed and approved by the local ethics committee.

\section{Consent for publication}

All patients had given their written consents.

\section{Author details}

${ }^{1}$ Radiology Department, Faculty of Medicine, Cairo University, Giza, Egypt.

${ }^{2}$ Faculty of Medicine, Cairo University, Giza, Egypt. ${ }^{3}$ General Surgery

Department, Faculty of Medicine, Cairo University, Giza, Egypt.

Received: 5 June 2019 Accepted: 30 July 2019

Published online: 19 September 2019

\section{References}

1. Moore KL, Agur AMR, Dalley AF (2011) Essential clinical anatomy, 5th edn. Lippincott Williams \& Wilkins, Baltimore, MD, pp 117-131

2. Drake RL, VogI W, Mitchell AWM, Gray H (2015) Gray's anatomy for students, 3rd edn. Elsevier/Churchill Livingstone, Philadelphia, pp 280-299

3. O'Rahilly, R. and Müller, F. Carpenter and Swenson (2008). Basic human anatomy: a regional study of human structure. W. B. Saunders Co., Philadelphia [online version, part 5 abdomen, chapter 25] Available at: https://www.dartmouth.edu/ humananatomy/ [Accessed 18 Jul. 2019].

4. Lee RK, Cho CC, Tong CS, Ng AW, Liu EK, Griffith JF (2013) Ultrasound of the abdominal wall and groin. Can Assoc Radiol J 64(4):295-305

5. Gokhale S (2007) High-resolution ultrasonography of the anterior abdominal wall. Indian J Radiol Imaging 17(4):290

6. Ahn SE, Park SJ, Moon SK, Lee DH, Lim JW (2016) Sonography of abdominal wall masses and masslike lesions. J Ultrasound Med 35(1):189-208

7. Jayaram P, Pereira F, Barrett J (2018) Evaluation of dynamic ultrasound scanning in the diagnosis of equivocal ventral hernias with surgical comparison. Br J Radiol 91:1088

8. Babu OS, Vidya NS (2018) High-resolution sonography in the evaluation of anterior abdominal wall lesions. Indian J App Res 7:8

9. Devareddy MM, Devakar S, Chetan M (2016) Evaluation the sonographic appearance of spectrum of anterior abdominal wall lesions and to compare the sonographic features with pathological and operative diagnosis: a crosssectional study. Int J Scientific Study 4(7):112-119

10. El-Mekkawy El-Sayed E (2015) Role of ultrasonography and multidetector $\mathrm{CT}$ in imaging of anterior abdominal wall lesions. Int J Medical Imaging 3(5):98

11. Lou L, Teng J, Qi H, Ban Y (2014) Sonographic appearances of desmoid tumors. J Ultrasound Medicine 33(8):1519-1525

\section{Publisher's Note}

Springer Nature remains neutral with regard to jurisdictional claims in published maps and institutional affiliations.

\section{Submit your manuscript to a SpringerOpen ${ }^{\circ}$ journal and benefit from:}

- Convenient online submission

- Rigorous peer review

- Open access: articles freely available online

- High visibility within the field

Retaining the copyright to your article

Submit your next manuscript at $>$ springeropen.com 